\title{
Hitting Time Analysis of OneMax Problem in Genetic Algorithm
}

\author{
Yifei Du \\ Graduate School of Engineering, University of Miyazaki, Gakuen Kibanadai-nishi 1-1 \\ Miyazaki City, Miyazaki Prefecture, 889-2192, Japan \\ QinLian Ma \\ Interdisciplinary Graduate School of Agriculture and Engineering, University of Miyazaki \\ Kenji Aoki \\ Information Technology Center, University of Miyazaki \\ Makoto Sakamoto and Hiroshi Furutani \\ Faculty of Engineering, University of Miyazaki \\ Yu-an Zhang \\ Department of Computer Science and Technology, Qinghai University, No. 251, Ning Road \\ Xining City, Qinghai Province, 810016, China \\ E-mail: furutani@cs.miyazaki-u.ac.jp
}

\begin{abstract}
Genetic algorithms (GAs) are stochastic optimization techniques, and we have studied the effects of stochastic fluctuation in the process of GA evolution. A mathematical study was carried out for GA on OneMax function within the framework of Markov chain model. We treated the task of estimating convergence time of the Markov chain for OneMax problem. Then, in order to study hitting time, we study the state after convergence.
\end{abstract}

Keywords: genetic algorithms, OneMax problem, Markov model, convergence time, hitting time

\section{Introduction}

Since GAs are stochastic optimization methods, we have to take into account a stochastic fluctuation to explain the behaviors of evolution. To do this, we made use of the Wright-Fisher model ${ }^{1}$, a type of Markov chain method. For example, we considered the effects of crossover on the evolution speed of OneMax problem², and carried out the microscopic investigation in terms of linkage analysis ${ }^{3}$. We also studied the convergence time of Markov chain in OneMax problem ${ }^{4}$. This analysis was performed by using the eigenvalues of transition matrix representing the behavior of population in the GA. From this analysis, we found that the convergence of GA to the stationary state can be represented approximately by mutation rate and string length. It is well known from the theory of finite Markov chain that ergodic Markov chain converges to the stationary distribution ${ }^{5}$. We obtained the approximate expression for predicting the convergence time to the stationary state in terms of one parameter.

In this paper, we report the hitting time analysis of GA by the use of Markov chain theory. The hitting time is the step at which the optimum solution appears in a population for the first time during the process of GA evolution. To simplify the analysis, we have separated the process into convergence time $T_{c}$ and hitting time after convergence $T_{h}$.

We estimate $T_{h}$ by experiments of OneMax function, and survey the impact of parameters on the hitting time after convergence. Our results demonstrate that the hitting time distribution $h(t)$ has an exponential form, 
and the logarithmic of $h(t)$ is linearly decreasing function. This means the distribution is almost determined by one parameter $b$. We estimate the value of $b$ from the experiments, and report the dependence of $b$ on the population size and mutation rate.

\section{Mathematical Model}

We treat the evolution process of a population with $N$ individuals. The individuals are represented by binary strings of length $l$, and there are $n=2^{l}$ genotypes,

$$
i=<i(l), \ldots, i(1)>, i(k) \in\{0,1\} \text {. }
$$

The OneMax fitness function $f_{i}$ is defined as

$$
f_{i}=\sum_{k=1}^{l} i(k) \text {. }
$$

Thus the string of all ones $\langle 1,1, \ldots, 1\rangle$ is the optimum solution of this function.

\section{Analysis of Hitting Time}

In our model, we will investigate the distribution of hitting time $H(t)$ approximately. Though it is difficult to obtain the theoretical expression of $H(t)$. Therefore, in order to study the hitting time $T_{H}$ directly, we have separated $T_{H}$ into converge time $T_{c}$ and hitting time after converge $T_{h}$. By analyzing $T_{c}$ and $T_{h}$, we can estimate the upper bound of hitting time ${ }^{1}$. So we assume

$$
T_{H} \leq T_{c}+T_{h}
$$

We have used the concept of Markov chain to analyze convergence time $T_{c}$. In this study, we used experimental method to estimate the hitting time after convergence $T_{h}$. In this study, we obtained the hitting time distribution after convergence $h(t)$ by the experiments.

\subsection{Analysis of convergence}

In our study, we used the Wright-Fisher model to theoretically analyze the process of population convergence. The Wright-Fisher model is one of the Markov model in population genetics ${ }^{6,7}$.

We consider the GA under positive mutation rate $p_{m}>0$. In this case, all elements of the transition matrix $P$ are positive, and the Markov chain of schema evolution is irreducible and aperiodic. The Markov chain theory states that an irreducible and aperiodic Markov chain converges to the stationary distribution $\pi$

$$
\lim _{t \rightarrow \infty} \mu(t)=\pi,
$$

and all elements $\pi_{i}$ are positive ${ }^{5}$.

In order to analyze the evolution of the first order schema, we referenced the total variation distance ${ }^{8}$. The total variation distance between the stationary distribution and the first order schema at generation $t$ is defined as

$$
T V(t)=\frac{1}{2} \sum_{i=0}^{N}\left|\mu_{i}(t)-\pi_{i}\right|
$$

By analyzing Markov chain, we have at large $t$

$$
T V(t)=C a^{t}
$$

where $C$ is a constant. It should be noted that the convergence behavior is determined by only one parameter $a$. The parameter $a$ is the second largest eigenvalue of the transition matrix ${ }^{1} P$.

$$
a=\left(1-\frac{1}{l}\right)\left(1-2 p_{m}\right)
$$

\subsection{Analysis of hitting time after convergence}

We obtained the hitting time distribution after convergence $h(t)$ by using the experiments of OneMax problem. The distribution $h(t)$ is the result of resetting generation $t=0$ when the population achieves the convergence state.

Our results demonstrate that the hitting time distribution $h(t)$ is an exponential form,

$$
h(t)=B(t)^{t},
$$

and the logarithmic of $h(t)$ is linearly decreasing function

$$
\log h(t)=t \log B(t)
$$

Then, we transform this equation to

$$
\log B(t)=\frac{\log h(t)}{t}
$$

We calculated the average of $\log B(t)$,

$$
\overline{\log B(t)}=\frac{\sum_{t} \log B(t)}{t},
$$

and defined a constant,

$$
b=\exp (\overline{\log B(t)}) .
$$

Thus the distribution is almost determined by $b$

$$
h(t) \approx b^{t}
$$

\section{Experiments}

We compared the theory with GA experiments. Crossover is uniform crossover with crossover rate $=1$. Mutation rate is $p_{m}$. Selection is roulette wheel selection. We averaged the results obtained by repeating 10000 calculations. 
total variation distance

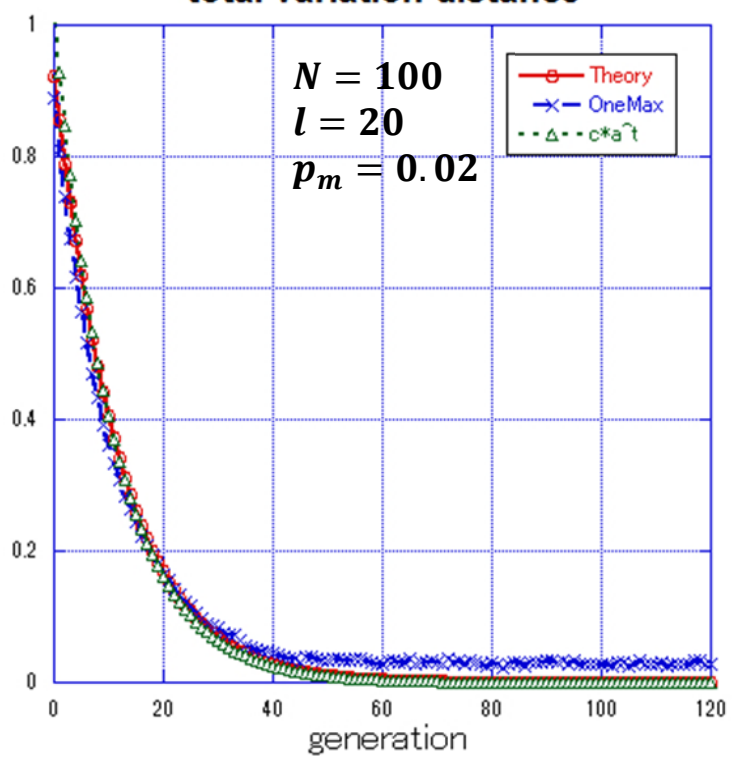

Fig. 1. Convergence of the first order schema

Figure 1 shows the results with $N=100, l=20$ and $p_{m}=0.02$. We used the matching time $t m=10$. The dotted line is the approximate curve obtained by eq.(6). We found that the convergence behavior of the GA calculation is well reproduced by eq.(6).

Figure 2 shows the results with $N=100$ and $l=20$.We used the total variation distance at generation 20 to show convergence rate. The line of "Theory" is the result of theoretical calculations. The line of "OneMax" is the result of experiments. We can know that at the same time the smaller the total variation distance is, the faster the convergence rate is. Therefore we can know the result of experiments consists with the result of theoretical calculations. The consistent result is that mutation rate is larger while convergence rate is faster.

Figure 3 shows the results with $N=100, l=20$ and $p_{m}=0.02$. The left figure is hitting time distribution $H(t)$. The right figure is hitting time distribution after convergence $h(t)$.

Figure 4 shows the results with $N=100, l=20$ and $p_{m}=0.02$. We calculated the results by eq.(10) and eq. (11).

Figure 5 shows the results with $N=100, l=20$ and $p_{m}=0.02$. The line of "hitting" is the distribution $h(t)$ is the result of resetting generation when the population achieves the convergence state. The line of " $b \wedge t$ " is the result of eq.(13). The two results are roughly consistent. So it means that the distribution is almost determined by one parameter $b$.

Figure 6 shows the results with $N=100$ and $l=20$. From the results we can know that the larger mutation rate is, the slower hitting time is.

\section{Summary}

We studied the hitting time of GA calculation using the fitness function of OneMax. We have separated hitting time into convergence time and hitting time after convergence. We analyzed the effects of mutation rate

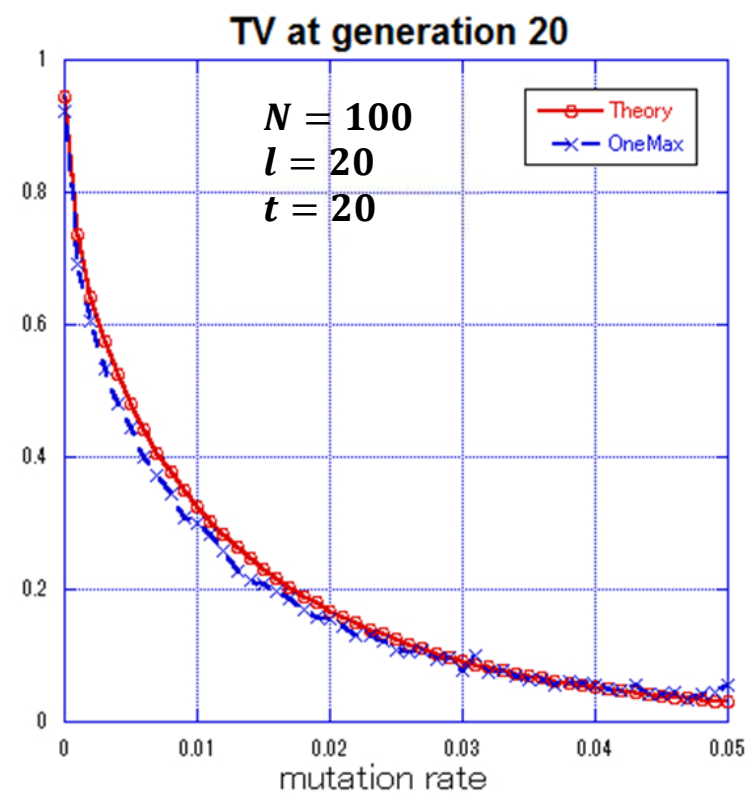

Fig. 2. Convergence of the first order schema at generation 20
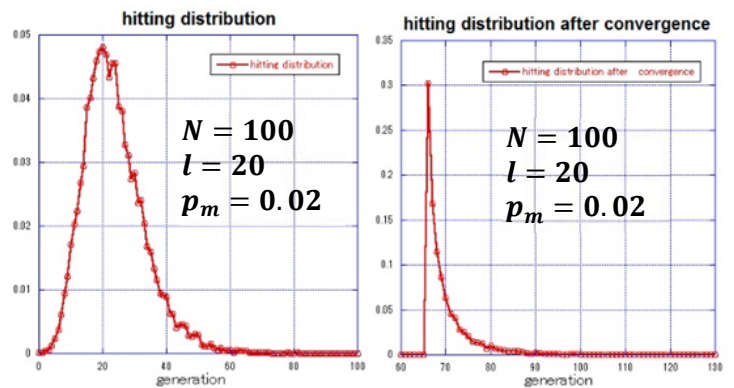

Fig. 3. Hitting distribution and hitting distribution after convergence 
$\log [h(t)]$

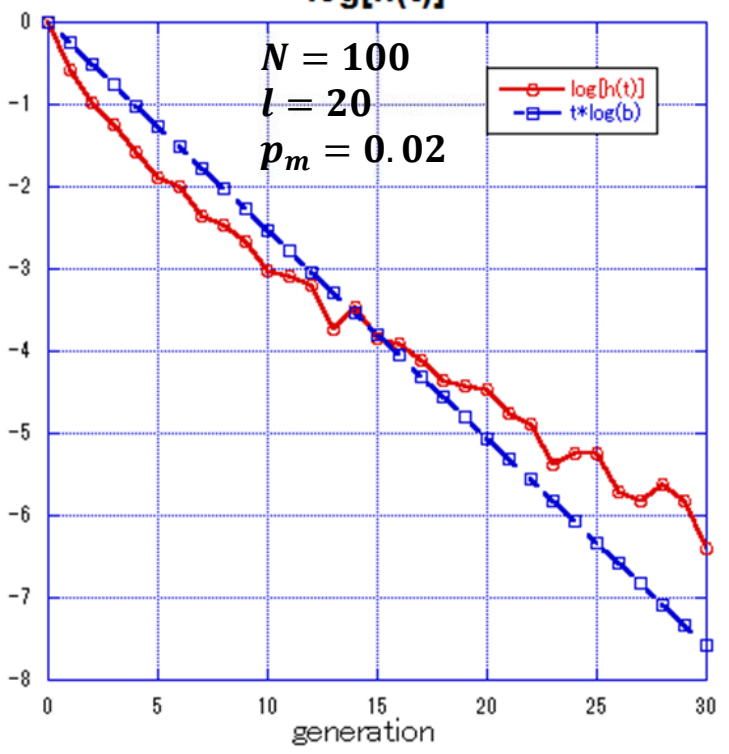

Fig. 4. $\log h(t)$ at generation
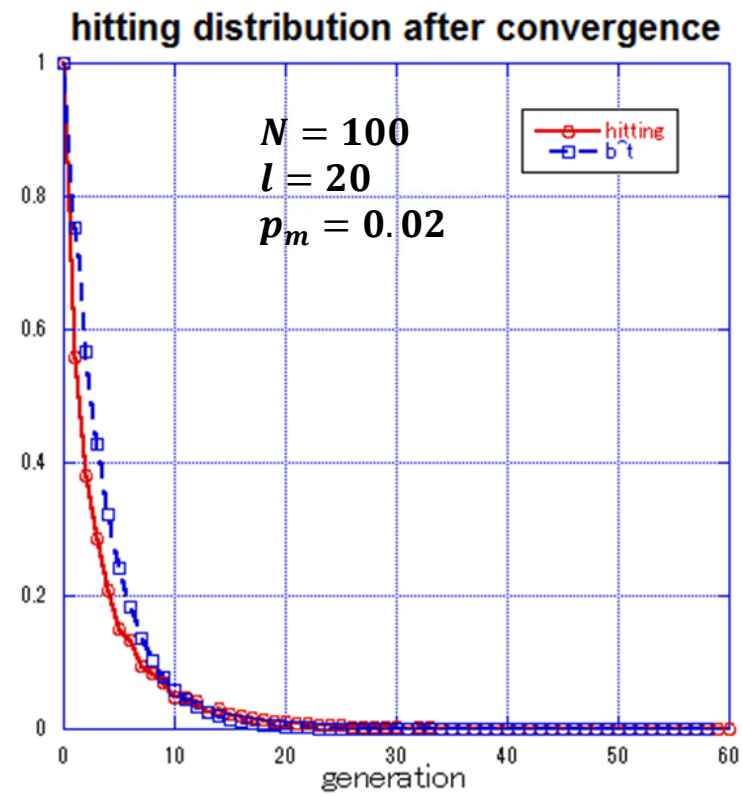

Fig. 5. Hitting distribution after convergence

on hitting time. In our previous results, we found that if mutation rate goes to large, convergence time becomes fast. In the present experiments, if mutation rate is larger, the hitting time after convergence becomes slower. Thus, we consider that there is a optimal value of mutation rate which can make the hitting time fastest. b

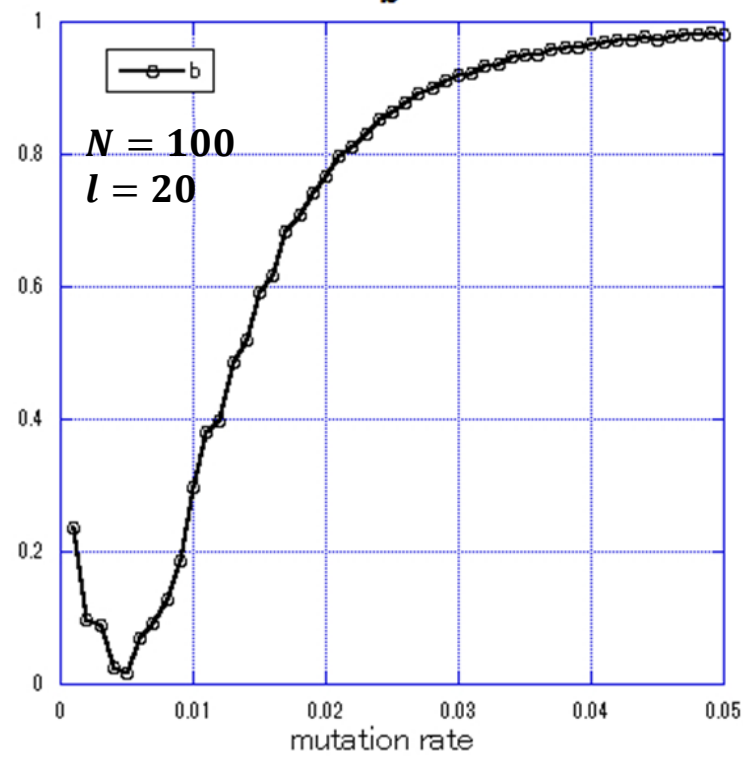

Fig. 6. The changes of $\boldsymbol{b}$ caused by mutation rate

\section{References}

1. H. Furutani, S. Katayama, M. Sakamoto, M. Ito (2007), Stochastic Analysis of Schema Distribution in a Multiplicative Landscape, Artificial Life and Robotics 11:101-104.

2. Y. Du, Q. Ma, Y. Zhang, M. Sakamoto and H. Furutani (2014), Runtime Analysis of OneMax Problem in Genetic Algorithm, Journal of Robotics, Networks and Artificial Life, Vol. 1, No. 3.

3. H. Furutani, Y. Zhang and M. Sakamoto (2009), Study of the Distribution of Optimum Solution in Genetic Algorithm by Markov Chains, IPSJ, Transactions on Mathematical Modeling and its Applications, 2:54-63.

4. H. Furutani (2003), Schema Analysis of OneMax Problem-Evolution Equation for First Order Schemata. in Foundations of Genetic Algorithms 7, Morgan Kaufmann, San Francisco, 9-26.

5. Q. Ma, Y. Zhang, K. Koga, K. Yamamori, M. Sakamoto and H. Furutani (2013), Stochastic analysis of OneMax problem by using Markov chain, Artificial Life and Robotics 17: 395-399.

6. J. W. J. Ewens (2004), Mathematical Population Genetics. I. Theoretical Introduction, Springer-Verlag, New York.

7. J. F. Crow and M. Kimura (1970), An Introduction to Population Genetics Theory, Harper and Row, New York.

8. D. A. Levin, Y. Peres and E. L. Wilmer (2008), Markov Chains and Mixing Times, American Mathematical Society, Providence. 